\title{
Effects of human serum on Balamuthia mandrillaris interactions with human brain microvascular endothelial cells
}

\author{
Abdul Matin, ${ }^{1}$ Seok Ryoul Jeong, ${ }^{1}$ Monique Stins ${ }^{2}$ \\ and Naveed Ahmed Khan ${ }^{1}$
${ }^{1}$ School of Biological and Chemical Sciences, Birkbeck College, University of London, London WC1E 7HX, UK USA \\ ${ }^{2}$ Pediatric Infectious Diseases, Johns Hopkins University School of Medicine, Baltimore, MD,
}

Correspondence

Noveed Ahmed Khan

n.khan@sbc.bbk.ac.uk

Received 20 July 2006

Accepted 11 September 2006
Balamuthia mandrillaris is a free-living amoeba and a causative agent of fatal granulomatous encephalitis. In the transmission of $B$. mandrillaris into the central nervous system (CNS), haematogenous spread is thought to be the primary step, followed by blood-brain barrier penetration. The objectives of the present study were (i) to determine the effects of serum from healthy individuals on the viability of $B$. mandrillaris, and (ii) to determine the effects of serum on $B$. mandrillaris-mediated blood-brain barrier perturbations. It was determined that normal human serum exhibited limited amoebicidal effects, i.e. $\sim 40 \%$ of trophozoites were killed. The residual subpopulation, although viable, remained static over longer incubations. Using human brain microvascular endothelial cells (HBMEC), which form the blood-brain barrier, it was observed that B. mandrillaris exhibited binding ( $>80 \%$ ) and cytotoxicity ( $>70 \%)$ to HBMEC. However, normal human serum exhibited more than $60 \%$ inhibition of $B$. mandrillaris binding and cytotoxicity to HBMEC. ELISAs showed that both serum and saliva samples exhibit the presence of anti- $B$. mandrillaris antibodies. Western blots revealed that normal human serum reacted with several $B$. mandrillaris antigens with approximate molecular masses of 148, 115, 82, 67, 60, 56, 44, 42, 40 and $37 \mathrm{kDa}$. Overall, the results demonstrated that normal human serum has inhibitory effects on $B$. mandrillaris growth and viability, as well as on their binding and subsequent cytotoxicity to HBMEC. A complete understanding of $B$. mandrillaris pathogenesis is crucial to develop therapeutic interventions and/or to design preventative measures.

\section{INTRODUCTION}

First discovered in 1986 by G. S. Visvesvara (Centers for Disease Control, USA), Balamuthia mandrillaris is an opportunistic protozoan pathogen that can cause fatal human infection involving the central nervous system (CNS) (Anzil et al., 1991; Taratuto et al., 1991; Visvesvara et al., 1990, 1993). Balamuthia granulomatous encephalitis (BGE) is characterized by headache, fever, characteristic skin lesions, stiff neck, nausea, vomiting, acute confused state, with cerebral haemorrhagic necrotizing lesions detected by neuroimaging scans, cranial nerve palsies, seizures and finally death (Jayasekera et al., 2004; reviewed by Schuster \& Visvesvara, 2004). The granuloma is composed of CD4-, CD8-positive $\mathrm{T}$ lymphocytes, B lymphocytes, plasma cells, multinucleate giant cells and macrophages, but it may be

Abbreviations: BGE, Balamuthia granulomatous encephalitis; CNS, central nervous system; HBMEC, human brain microvascular endothelial cells; LDH, lactate dehydrogenase; slgA, secretory $\lg \mathrm{A}$. absent in severely immunocompromised patients (Schuster \& Visvesvara, 2004). Although the predisposing factors in contracting BGE are not known, recent studies have shown that, unlike Acanthamoeba, B. mandrillaris can cause fatal infections in relatively immunocompetent people. This is shown by the findings that BGE can develop in patients with no history of syphilis, diabetes mellitus, malignancies, or fungal and mycobacterial infections, and who are negative for HIV-1 and HIV-2. The portal of entry into the CNS is thought to be the olfactory neuroepithelium (Kiederlan \& Laube, 2004) or lower respiratory tract, followed by haematogenous spread. Skin lesions may provide direct entry into the bloodstream, bypassing the lower respiratory tract. In the case of haematogenous spread, amoebae entry into the CNS most likely occurs at the blood-brain barrier (Martinez et al., 2001; Schuster \& Visvesvara, 2004). Infection of the skin and lungs can last for months, but the involvement of the CNS results in death within days (Jayasekera et al., 2004). Recent studies have shown that $B$. mandrillaris exhibits multifactorial properties to produce 
damage of human brain microvascular endothelial cells (HBMEC), which form the blood-brain barrier (Jayasekera et al., 2004, 2005; Matin et al., 2006). However, the effects of normal human serum on $B$. mandrillaris interactions with HBMEC are not known and are the subject of the present study.

\section{METHODS}

HBMEC cultures. Primary HBMEC were obtained and cultured as previously described (Alsam et al., 2003; Stins et al., 1997). Briefly, HBMEC were routinely grown in $20 \%$ heat-inactivated fetal bovine serum, $2 \mathrm{mM}$ glutamine, $1 \mathrm{mM}$ pyruvate, $100 \mathrm{U}$ penicillin $\mathrm{ml}^{-1}$, $100 \mu \mathrm{g}$ streptomycin $\mathrm{ml}^{-1}$, non-essential amino acids, vitamins and RPMI 1640 (Invitrogen). For experiments, HBMEC were grown in 24 -well plates by inoculating $5 \times 10^{5}$ cells in $1 \mathrm{ml}$ into each well, and plates were incubated at $37^{\circ} \mathrm{C}$ in a $5 \% \mathrm{CO}_{2}$ incubator. At this cell density, confluent monolayers were formed within $24 \mathrm{~h}$, and these were used for subsequent experiments.

Cultures of B. mandrillaris. B. mandrillaris isolated from the brain of a mandrill baboon was obtained from the American Type Culture Collection (ATCC 50209). B. mandrillaris were routinely cultured on HBMEC as a food source, as previously described (Jayasekera et al., 2004; Matin et al., 2006). Briefly, B. mandrillaris were inoculated $\left(10^{6}\right.$ parasites in $10 \mathrm{ml}$ RPMI 1640) on HBMEC monolayers grown in T-75 tissue-culture flasks. B. mandrillaris consumed the HBMEC monolayer within $48 \mathrm{~h}$, and produced approximately $5-8 \times 10^{6}$ parasites per $10 \mathrm{ml}$ ( $>99 \%$ in trophozoite forms), which were used for subsequent experiments.

Adhesion assays. To determine whether normal human serum affects $B$. mandrillaris adhesion to HBMEC, adhesion assays were performed (Sissons et al., 2005). Briefly, HBMEC were grown to confluency in 24 -well plates. B. mandrillaris $\left(2 \times 10^{5}\right.$ amoebae $)$ were pre-incubated with 20 and $100 \%$ human serum (obtained from healthy individuals by Harlan SeraLab, tested negative for hepatitis $C$ virus, HIV-1, HIV-2 and hepatitis B surface antigen, and considered to be normal human serum). When purchased, each batch of serum was from one individual, and several batches were obtained. To test the involvement of complement pathways, assays were performed using heat-inactivated serum $\left(56^{\circ} \mathrm{C}\right.$ for $30 \mathrm{~min}$ to inactivate complement components) as previously described (Toney \& Marciano-Cabral, 1998). Next, amoebae plus serum were transferred to HBMEC monolayers in 24-well plates, and the plates were incubated at $37^{\circ} \mathrm{C}$ in $5 \% \mathrm{CO}_{2}$ for $60 \mathrm{~min}$. After this incubation, the unbound amoebae were counted using a haemocytometer, and the percentage of unbound amoebae was calculated from the following equation: (number unbound amoebae/total number amoebae) $\times 100$. The percentage of bound amoebae was calculated from the following equation: $100-$ percentage of unbound amoebae.

Cytotoxicity assays. Cytotoxicity assays were performed as previously described (Alsam et al., 2003). Briefly, B. mandrillaris with or without human serum was incubated with HBMEC monolayers grown in 24-well plates, as described for adhesion assays. Plates were incubated at $37^{\circ} \mathrm{C}$ in a $5 \% \mathrm{CO}_{2}$ incubator and periodically observed for cytopathic effects for up to $24 \mathrm{~h}$. At the end of this incubation period, supernatants were collected and cytotoxicity was determined by measuring lactate dehydrogenase (LDH) release (cytotoxicity detection kit; Roche Applied Science). Briefly, conditioned media of co-cultures of amoebae and HBMEC were collected, and the percentage cytotoxicity was calculated as follows: ((sample value-control value $) /($ total $\mathrm{LDH}$ release - control value $)(\times 100$. Control values were obtained from HBMEC incubated in RPMI alone. Total LDH release was determined from HBMEC treated with $1 \%$ Triton X-100 for $30 \mathrm{~min}$ at $37^{\circ} \mathrm{C}$.

Amoebicidal and amoebistatic assays. For amoebicidal assays, B. mandrillaris trophozoites were incubated with 20 and $100 \%$ normal human serum and heat-inactivated serum, as described above. The numbers of $B$. mandrillaris at various time intervals were determined using haemocytometer counting. The counts from $B$. mandrillaris incubated alone in the absence of serum were taken as $100 \%$, and results are presented as a percentage relative to that value. For amoebistatic assays, B. mandrillaris trophozoites were added to HBMEC in the presence of normal human serum and heat-inactivated serum. At various time intervals, B. mandrillaris were washed from the surface of HBMEC monolayers and numbers were determined by haemocytometer counting. The counts from $B$. mandrillaris plus HBMEC in the absence of serum were taken as $100 \%$, and results are presented as a percentage relative to that value.

Western blots. The presence of anti-B. mandrillaris IgG in human serum was determined by Western blots, as previously described (Khan et al., 2002). Briefly, various numbers of B. mandrillaris were mixed (1:1) with sample buffer containing $10 \% \beta$-mercaptoethanol and heated at $100{ }^{\circ} \mathrm{C}$ for $5 \mathrm{~min}$. Samples were centrifuged at $10000 \mathrm{~g}$ for $1 \mathrm{~min}$ and electrophoresed by $12.5 \%$ SDS-PAGE. Proteins were transferred onto nitrocellulose membranes. The mem-

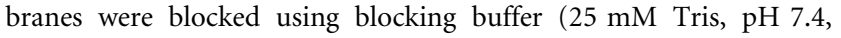
$150 \mathrm{mM} \mathrm{NaCl}, 0.1 \%$ Tween-20) containing $4 \%$ skimmed milk for $60 \mathrm{~min}$ at $22^{\circ} \mathrm{C}$. After this incubation, the membranes were immunoblotted using normal human serum $(1 \mathrm{ml})$ overnight at $4{ }^{\circ} \mathrm{C}$. Next day, membranes were washed three times with PBS and incubated with an appropriate horseradish peroxidase-linked secondary antibody for $1 \mathrm{~h}$. Finally, blots were washed and developed using an enhanced chemiluminescence kit (Amersham Biosciences).

ELISAs. The presence of anti-B. mandrillaris IgG in normal human serum and secretory $\operatorname{IgA}$ ( $\operatorname{sgA}$ ) in mucosal secretions (saliva was obtained from healthy individuals) was determined using ELISAs, as previously described (Leher et al., 1998). Briefly, B. mandrillaris were added to 96-well plates $\left(2 \times 10^{5}\right.$ amoebae per well). Subsequently, wells were air-dried, followed by the addition of icecold methanol and acetone $(1: 1)$ for $45 \mathrm{~min}$. The wells were washed twice with PBS containing $0.05 \%$ Tween-20 to remove non-adherent amoebae, and blocked using $3 \%$ BSA for $1 \mathrm{~h}$ at $37^{\circ} \mathrm{C}$. The normal human serum and saliva samples were serially diluted from $1: 1$ to $1: 50000$. B. mandrillaris were washed and $100 \mu$ of test samples was added to the wells and incubated for $18 \mathrm{~h}$ at $4{ }^{\circ} \mathrm{C}$. The following day, amoebae were washed five times with PBS plus Tween-20 and incubated with the respective secondary antibody. For B. mandrillaris incubated with saliva samples, wells were incubated with mouse anti-human IgA (Abcam) for $60 \mathrm{~min}$ and washed five times, as above. For B. mandrillaris incubated with serum, this step was omitted. Next, anti-mouse IgG antibody conjugated to horseradish peroxidase was added to all wells. The plates were incubated for $1 \mathrm{~h}$ at $37^{\circ} \mathrm{C}$. Finally, the wells were washed five times as above and $100 \mu \mathrm{l}$ substrate solution $(0.1 \%$ $\mathrm{H}_{2} \mathrm{O}_{2}, 0.1 \%$ orthophenylenediamine in citrate buffer) was added. The reactions were allowed to develop for $15 \mathrm{~min}$, and finally $100 \mu \mathrm{l}$ $3 \%$ sulphuric acid was added to stop the reaction. The $A_{492}$ of each well was determined on a microplate reader (Anthos 2020, JenconsPLS). The $A_{492}$ of $B$. mandrillaris incubated with secondary antibody in the absence of serum and saliva samples was taken as the background. 


\section{RESULTS AND DISCUSSION}

\section{Serum from healthy individuals inhibits B. mandrillaris adhesion to HBMEC}

To determine the effects of normal human serum on $B$. mandrillaris binding to HBMEC, adhesion assays were performed. We observed that serum inhibited up to $50 \%$ of amoeba binding to HBMEC monolayers (Fig. 1). Next, to determine the effects of heat inactivation on the inhibitory effects of serum, adhesion assays were performed using heatinactivated serum. The results revealed that normal and heat-inactivated serum exhibited similar inhibitory effects on amoeba binding to HBMEC (Fig. 1).

\section{Serum from healthy individuals inhibits $B$. mandrillaris-mediated HBMEC cytotoxicity}

To determine the effects of normal human serum on $B$. mandrillaris-mediated HBMEC death, cytotoxicity assays were performed. In the absence of serum, B. mandrillaris produced severe HBMEC cell cytotoxicity (more than $70 \%$ ) within $24 \mathrm{~h}$ (Fig. 2). However, B. mandrillaris-mediated HBMEC cytotoxicity was inhibited in the presence of serum (Fig. 2). Again, heat-inactivation of serum did not abolish serum effects (Fig. 2). Overall, these data show that human serum partially inhibits $B$. mandrillaris-mediated HBMEC cytotoxicity.

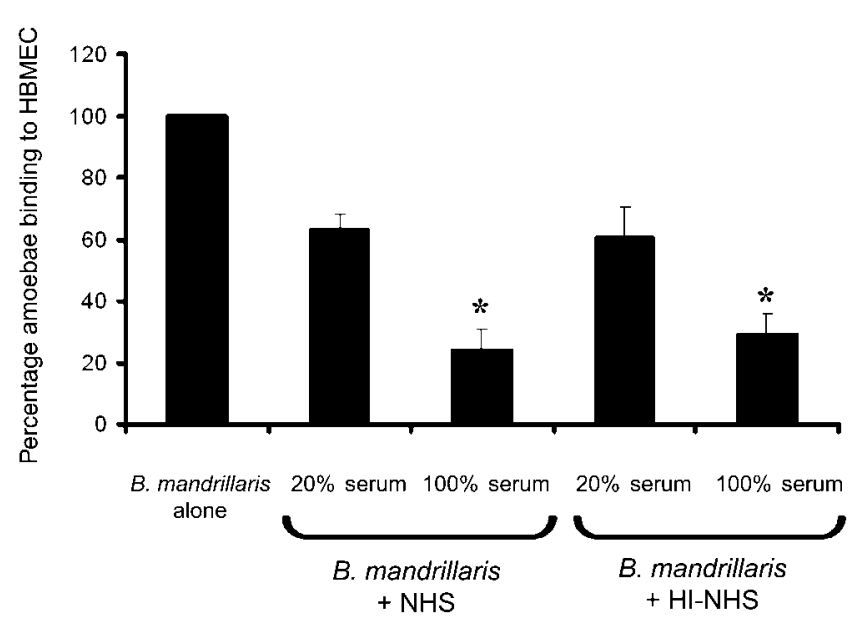

Fig. 1. Serum from healthy individuals inhibits $B$. mandrillaris adhesion to HBMEC. HBMEC were grown to monolayers in 24-well plates and incubated with $2 \times 10^{5} \mathrm{~B}$. mandrillaris for $1 \mathrm{~h}$ at $37^{\circ} \mathrm{C}$ in the presence of 20 or $100 \%$ normal human serum (NHS) or heat-inactivated serum (HI-NHS). Note that serum inhibited $B$. mandrillaris adhesion to HBMEC in a heatinactivation-independent manner $\left({ }^{\star} P<0.05\right.$ using Student's $t$ test with paired, two-tailed distribution). Results are representative of three independent experiments performed in triplicate; bars represent standard error.

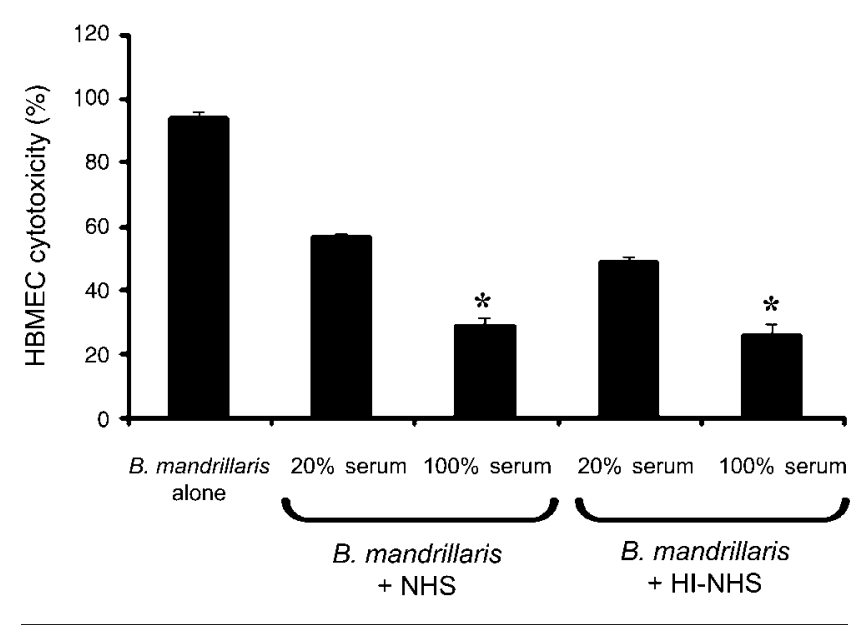

Fig. 2. Serum from healthy individuals inhibits $B$. mandrillarismediated HBMEC cytotoxicity. B. mandrillaris were added to HBMEC monolayers in 24-well plates and incubated at $37^{\circ} \mathrm{C}$ for up to $24 \mathrm{~h}$. HBMEC without $B$. mandrillaris were used as controls. The percentage HBMEC cytotoxicity was determined by measuring $\mathrm{LDH}$ release, as described in Methods. Serum inhibits $B$. mandrillaris-mediated HBMEC cytotoxicity in a heatinactivation-independent manner $\left({ }^{*} P<0.05\right.$ using Student's $t$ test with paired, two-tailed distribution). Results are representative of three independent experiments performed in triplicate; bars represent standard error.

\section{Serum from healthy individuals induces an initial amoebicidal effect followed by amoebistatic activity}

To determine whether the effects of serum on $B$. mandrillaris virulence properties are mediated via distinct molecular mechanisms or are simply secondary to the amoebistatic/ amoebicidal properties of serum, assays were performed as described in Methods. Our findings revealed that $100 \%$ serum exhibited partial amoebicidal effects, with an initial reduction in amoeba numbers (Fig. 3). However, a subpopulation of $B$. mandrillaris remained intact. In the presence of $100 \%$ serum, cultures remained static over longer incubations (Fig. 4). However, in the presence of $20 \%$ serum, a non-significant increase $(P>0.05)$ in the numbers of $B$. mandrillaris was observed. Furthermore, to determine whether this subpopulation of $B$. mandrillaris was viable, $50 \mu \mathrm{l}$ culture suspension was inoculated onto HBMEC monolayers, and their viability was determined by Trypan blue exclusion assay. B. mandrillaris were negative for Trypan blue and appeared as viable trophozoites on HBMEC monolayers, suggesting that the effect of serum on the remaining population was amoebistatic (data not shown).

\section{ELISA demonstrates the presence of $B$. mandrillaris-specific IgG in serum and slgA in mucosal secretions}

To determine the presence of anti-B. mandrillaris IgG in normal human serum and SIgA in saliva, ELISAs were 


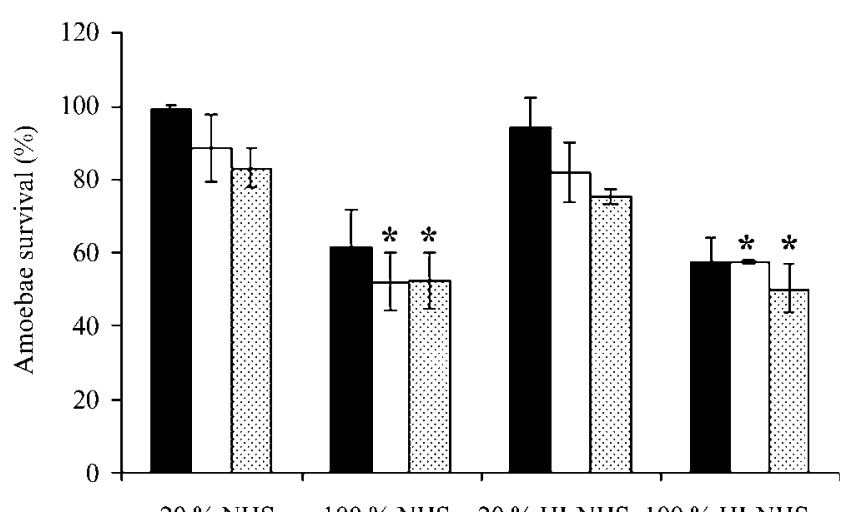

$20 \%$ NHS $\quad 100 \%$ NHS $20 \%$ HI-NHS $100 \%$ HI-NHS

Fig. 3. Serum exhibits amoebicidal effects. B. mandrillaris were incubated in the presence of 20 and $100 \%$ normal human serum (NHS) or heat-inactivated serum (HI-NHS) for different lengths of time: black bars, $30 \mathrm{~min}$; white bars, $1 \mathrm{~h}$; grey bars, $24 \mathrm{~h}$. Cell numbers and their viability were determined by haemocytometer counting and the Trypan blue exclusion assay. The counts from $B$. mandrillaris incubated alone in the absence of serum were taken as $100 \%$, and results are presented as a percentage relative to that value. Note that $100 \%$ serum exhibited an initial amoebicidal effect, followed by an amoebistatic effect $\left({ }^{\star} P<0.05\right.$ using Student's $t$ test with paired, two-tailed distribution). Results are representative of three independent experiments performed in triplicate; bars represent standard error.

performed. As shown in Fig. 5, serum and saliva exhibited the presence of anti-B. mandrillaris IgG and sIgA, respectively. Serum and saliva samples reacted specifically with B. mandrillaris in a dose-dependent manner (Fig. 5). Samples from three different individuals were tested and exhibited similar results (representative data are shown in Fig. 5).

\section{Western blots reveal that serum antibodies react with several $B$. mandrillaris antigens}

Earlier studies have shown that normal human serum possesses anti-B. mandrillaris antibodies (Huang et al., 1999). To determine whether human serum possesses antibodies that react with $B$. mandrillaris antigens, Western blotting assays were performed. The findings revealed that normal human serum reacted with several $B$. mandrillaris antigens with approximate molecular masses of 148, 115, 82, 67, 60, 56, 44, 42, 40 and $37 \mathrm{kDa}$ (Fig. 6).

With the growing HIV pandemic, it is reasonable to predict increasing numbers of opportunistic infections. This is particularly worrying in developing countries where HIV patients have no or limited access to novel antiretroviral therapies. Thus there is a need for continued efforts to (i) increase awareness, (ii) develop rapid diagnostic methods and (iii) understand the basic molecular mechanisms of host-parasite interactions, which should help design preventative and/or therapeutic strategies. Among

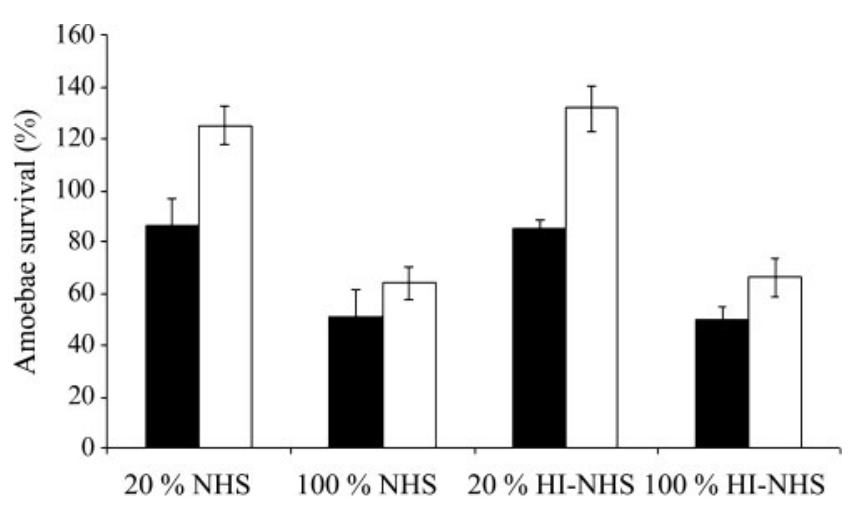

Fig. 4. Serum exhibits amoebistatic effects. B. mandrillaris were incubated with HBMEC (as food source) in the presence of 20 or $100 \%$ human serum for different lengths of time: black bars, 1 h; white bars, 24 h. As indicated in Fig. 3, 100\% serum exhibited an initial amoebicidal effect. However, the remaining subpopulation of $B$. mandrillaris remained static over longer incubations, even in the presence of a food source, i.e. HBMEC. The counts from B. mandrillaris incubated alone in the absence of serum and HBMEC were taken as $100 \%$, and results are presented as a percentage relative to that value. Note that $100 \%$ serum exhibited an initial amoebicidal effect, followed by an amoebistatic effect. Results are representative of three independent experiments performed in triplicate; bars represent standard error.

opportunistic protozoan pathogens, B. mandrillaris has gained particular attention in recent years. This is due to its ability to produce BGE in both immunocompromised and immunocompetent individuals. One of the major steps in BGE is amoebae invasion of the bloodstream followed by

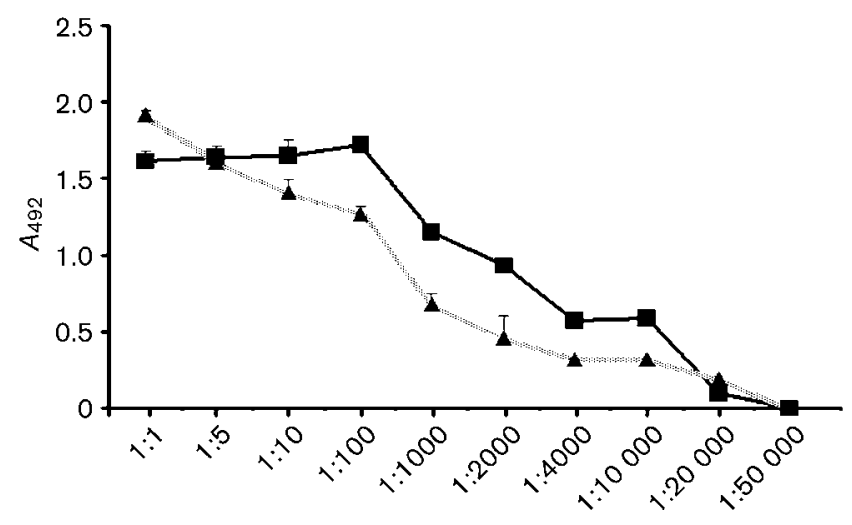

Fig. 5. Serum contains B. mandrillaris-specific lgG. To determine whether serum contained $B$. mandrillaris-specific $\lg$, ELISAs were performed as described in Methods. Serum (ם) and saliva $(\boldsymbol{\Delta})$ samples contained $B$. mandrillaris-specific $\lg G$ and $\operatorname{sig} A$, respectively, in a dose-dependent manner, and the levels of $B$. mandrillaris-specific antibodies were similar. Results are means of three independent experiments performed in duplicate; bars represent standard error. 


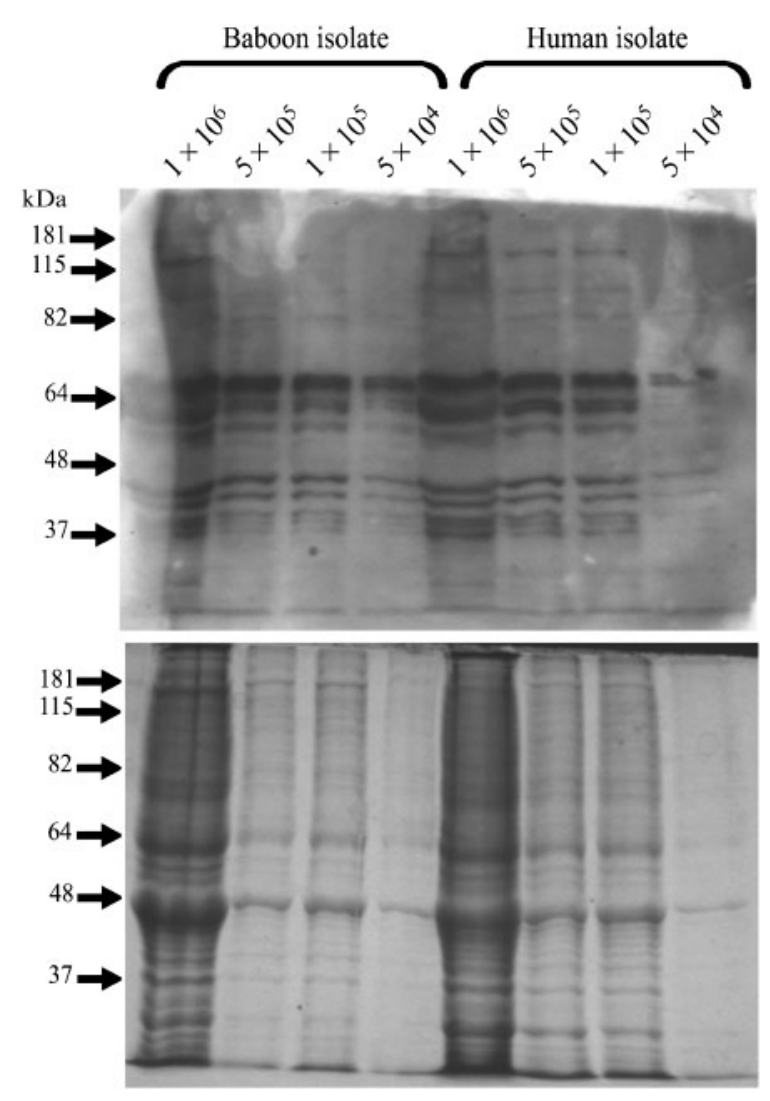

Fig. 6. Serum reacts with several antigens of $B$. mandrillaris. To determine whether normal human serum possesses antibodies which react with $B$. mandrillaris antigens, Western blots (upper panel) were performed as described in Methods. Our findings revealed that normal human serum reacted with $B$. mandrillaris antigens with approximate molecular masses of 148, 115, 82, 67, 60, 56, 44, 42, 40 and $37 \mathrm{kDa}$ (Fig. 6). Lower panel, SDS-PAGE.

their haematogenous spread. B. mandrillaris entry into the CNS most likely occurs at the blood-brain barrier. Here, we studied the effects of serum on $B$. mandrillaris interactions with HBMEC, which form the blood-brain barrier. To the best of our knowledge, our findings reveal for the first time that normal human serum inhibits $B$. mandrillaris binding and subsequent cytotoxicity to HBMEC. Overall, these studies suggest that normal human serum protects HBMEC against $B$. mandrillaris, and that this property may help prevent blood-brain barrier perturbations.

Next, we determined whether the protective effects of serum against $B$. mandrillaris are targeted via distinct molecular mechanisms or are simply an effect secondary to the amoebistatic/amoebicidal properties of serum. We observed that normal human serum exhibited an initial limited amoebicidal effect: $\sim 40 \%$ of trophozoites were killed. However, a subpopulation of amoebae remained viable, although cultures were stationary over longer incubations.
The fact that serum exhibited $\sim 50 \%$ inhibition of amoebae binding to HBMEC (similar to amoebicidal effects) suggests that the effects of serum on the properties of $B$. mandrillaris are at least partly secondary to the amoebicidal/amoebistatic effects. This is consistent with earlier findings, which show that virulent strains of Acanthamoeba (a close relative of $B$. mandrillaris) resist serum-mediated killing (Toney \& Marciano-Cabral, 1998).

Although BGE has been reported in immunocompetent populations (individuals who are negative for syphilis, diabetes mellitus and malignancies, and fungal, HIV-1, HIV-2 and mycobacterial infections, as well as possessing normal CD4- and CD8-positive T-lymphocyte and Blymphocytes counts), given the rarity of the disease, it is hard to imagine that there are no predisposing factors in contracting BGE. But whether the predisposing factors are other primary infections, underlying genetic factors, exposure to an environment with widely distributed $B$. mandrillaris or a combination of the above is incompletely understood. For example, most BGE cases have occurred in the Americas (more than $90 \%$ of cases), with the majority reported from warmer regions (Schuster \& Visvesvara, 2004). Among them, a significant number of BGE cases have occurred in individuals of Hispanic origin (Schuster et al., 2004). Future studies will aim to differentiate between the possibility that individuals of Hispanic origin are more exposed to B. mandrillaris and the possibility that they have a genetic predisposition to succumb to this disease, as suggested by Schuster et al. (2004).

One of the interesting findings in this study was that serum possesses antibodies that react with several $B$. mandrillaris antigens in Western blots. The antigens of $B$. mandrillaris reacted strongly with normal human serum. B. mandrillaris isolates from baboon tissue (ATCC 50209) and from human brain (Jayasekera et al., 2005) shared several common antigens, which confirms that the two isolates are antigenically close and belong to the same species. However, the protective role of antibodies against BGE is somewhat unclear. For example, several BGE patients have been reported to possess high titres of anti- $B$. mandrillaris antibodies without a protective response, resulting in death (Huang et al., 1999; Jayasekera et al., 2004). This may be due to a delayed humoral response, overwhelming BGE infection, or the ability of amoebae to evade the humoral immune response. Overall, these findings confirm that normal human serum is partially inhibitory to $B$. mandrillaris properties associated with pathogenesis, but whether a healthy immune response is sufficient to control and/or eradicate these life-threatening pathogens is unknown. To this end, studies are being conducted to determine the detrimental effects of serum on B. mandrillaris in the presence of neutrophils/ macrophages. These studies should clarify the mechanisms associated with B. mandrillaris pathogenesis, and this may help design preventative measures and/or develop therapeutic interventions. 


\section{ACKNOWLEDGEMENTS}

This work was partially supported by grants from the Faculty Research Fund and Central Research Fund, University of London.

\section{REFERENCES}

Alsam, S., Kim, K. S., Stins, M., Rivas, A. O., Sissons, J. \& Khan, N. A. (2003). Acanthamoeba interactions with human brain microvascular endothelial cells. Microb Pathog 35, 235-241.

Anzil, A. P., Rao, C., Wrzolek, M. A., Visvesvara, G. S., Sher, J. H. \& Kozlowski, P. B. (1991). Amebic meningoencephalitis in a patient with AIDS caused by a newly recognized opportunistic pathogen. Leptomyxid ameba. Arch Pathol Lab Med 115, 21-25.

Huang, Z. H., Ferrante, A. \& Carter, R. F. (1999). Serum antibodies to Balamuthia mandrillaris, a free-living amoeba recently demonstrated to cause granulomatous amoebic encephalitis. J Infect Dis 179, 1305-1308.

Jayasekera, S., Sissons, J., Tucker, J. Rogers C., Nolder, D., Warhurst, D., Alsam, S., White, J. M. L., Higgins, E. M. \& Khan, N. A. (2004). Post-mortem culture of Balamuthia mandrillaris from the brain and cerebrospinal fluid of a case of granulomatous amoebic meningoencephalitis, using human brain microvascular endothelial cells. J Med Microbiol 53, 1007-1012.

Jayasekera, S., Matin, A., Sissons, J., Maghsood, A. H. \& Khan, N. A. (2005). Balamuthia mandrillaris stimulates IL-6 release from primary human brain microvascular endothelial cells via a phosphatidylinositol 3-kinase-dependent pathway. Microb Infect 7, 1345-1351.

Khan, N. A., Jarroll, E. L. \& Paget, T. A. (2002). Molecular and physiological differentiation between pathogenic and nonpathogenic Acanthamoeba. Curr Microbiol 45, 197-202.

Kiederlan, A. F. \& Laube, U. (2004). Balamuthia mandrillaris, an opportunistic agent of granulomatous amebic encephalitis, infects the brain via the olfactory nerve pathway. Parasitol Res 94, 49-52.
Matin, A., Stins, M., Kim, K. S. \& Khan, N. A. (2006). Balamuthia mandrillaris exhibits metalloprotease activities. FEMS Immunol Med Microbiol 47, 83-91.

Martinez, A. J., Schuster, F. L. \& Visvesvara, G. S. (2001). Balamuthia mandrillaris: its pathogenic potential. J Eukaryot Microbiol Suppl, 6S-9S.

Leher, H. F., Alizadeh, H., Taylor, W. M., Shea, A. S., Silvany, R. S., Van Klink, F., Jager, M. J. \& Niederkorn, J. Y. (1998). Role of mucosal $\operatorname{IgA}$ in the resistance to Acanthamoeba keratitis. Invest Ophthalmol Vis Sci 39, 2666-2673.

Schuster, F. L. \& Visvesvara, G. S. (2004). Free-living amoebae as opportunistic and non-opportunistic pathogens of humans and animals. Int J Parasitol 34, 1001-1027.

Schuster, F. L., Glaser, C., Honarmand, S., Maguire, J. H. \& Visvesvara, G. S. (2004). Balamuthia amebic encephalitis risk, Hispanic Americans. Emerg Infect Dis, 2004 Aug. http:// www.cdc.gov/ncidod/eid/vol10no8/04-0139.htm

Sissons, J., Kim, K. S., Stins, M., Jayasekera, S., Alsam, S. \& Khan, N. A. (2005). Acanthamoeba induces host cell death via a phosphatidylinositol 3-kinase (PI3K)-dependent mechanism. Infect Immun 73, 2704-2708.

Stins, M. F., Gilles, F. \& Kim, K. S. (1997). Selective expression of adhesion molecules on human brain microvascular endothelial cells. J Neuroimmunol 76, 81-90.

Taratuto, A. L., Monges, J., Acefe, J. C., Meli, F., Parades, A. \& Martinez, A. J. (1991). Leptomyxid amoeba encephalitis: report of the first case in Argentina. Trans R Soc Trop Med Hyg 85, 77.

Toney, D. M. \& Marciano-Cabral, F. (1998). Resistance of Acanthamoeba species to complement lysis. J Parasitol 84, 338-344.

Visvesvara, G. S., Martinez, A. J., Schuster, F. L., Leitch, G. J., Wallace, S. V., Sawyer, T. K. \& Anderson, M. (1990). Leptomyxid ameba, a new agent of amebic meningoencephalitis in humans and animals. J Clin Microbiol 28, 2750-2756.

Visvesvara, G. S., Schuster, F. L. \& Martinez, A. J. (1993). Balamuthia mandrillaris, N. G., N. Sp., agent of amebic meningoencephalitis in humans and other animals. J Eukaryot Microbiol 40, 504-514. 